\title{
Degree of Mucosal Damage and Clinical Presentation of Celiac Disease - Is There a Connection?
}

\author{
Pavlovic Momcilo, ${ }^{1, *}$, Berenji Karolina ${ }^{1}$, Bukurov Marko ${ }^{1}$, Radlovic Nedeljko ${ }^{2}$, Lekovic Zoran ${ }^{2}$, \\ Stojsic Zorica ${ }^{3}$, Novak Arpad ${ }^{4}$, Rokvic Zeljko ${ }^{4}$, Arsic Bogdan ${ }^{4}$ \\ ${ }^{1}$ College of Vocational Studies in Subotica, Banijska 67, 24000 Subotica, Serbia \\ ${ }^{2}$ Department of Gastroenterology, University Children Hospital, Tirsova 10, 11000 Belgrade, Serbia \\ ${ }^{3}$ Institute of Pathology, Dr Subotica 1, 11000 Belgrade, Serbia \\ ${ }^{4}$ General Hospital Subotica, Izvorska 3, 24000 Subotica, Serbia \\ *Corresponding author: momodec@tippnet.rs
}

\begin{abstract}
Celiac disease (CD) is an immune-mediated enteropathy induced by gliadin and related prolamins. This study was designed to evaluate the correlation between the degree and uniformity of mucosal damage and forms of CD. The study included a total of 85 children (33 boys and 52 girls, mean age 6.59 years) hospitalized due to CD. Patients were assigned to one of three groups based on their form of disease manifestation: (1) patients with classical form; (2) patients with atypical form; and (3) patients with asymptomatic celiac disease. The diagnosis of CD was based on positive CD specific antibodies, HLA typing and histological analysis of multiple biopsy samples. Histological changes were classified using modified Marsh criteria. Forty one patients had classical form, 32 had atypical form, while 12 patients had asymptomatic form of celiac disease. There was no difference in the degree of damage on small bowel samples between different clinical forms of celiac disease $(\mathrm{p}=0.079)$. The frequency of uniformity of enteropathy does not depend on clinical form of the disease $(\mathrm{p}=0.882)$. Uniform small bowel mucosal damage was more common in cases of severe degree of mucosal damage $(\mathrm{p}=0.017)$. The degree of damage and uniformity of changes in duodenal biopsy specimens did not correlate with mode of presentation, while uniform mucosal damage increased with severity of enteropathy.
\end{abstract}

Keywords: celiac disease, clinical presentation, duodenal biopsy, marsh classification, villous atrophy

Cite This Article: Pavlovic Momcilo, Berenji Karolina, Bukurov Marko, Radlovic Nedeljko, Lekovic Zoran, Stojsic Zorica, Novak Arpad, Rokvic Zeljko, and Arsic Bogdan, "Degree of Mucosal Damage and Clinical Presentation of Celiac Disease - Is There a Connection?.” International Journal of Celiac Disease, vol. 4, no. 1 (2016): 11-15. doi: 10.12691/ijcd-4-1-9.

\section{Introduction}

Celiac disease (CD) is an autoimmune disorder caused by permanent form of intolerance to gluten, i.e. gliadin and related prolamins from seeds of wheat, rye and barley [1]. Based on its primary feature, it belongs to a group of multifactorial diseases, i.e. it develops as a result of an inherited (polygenic) disposition and exposure to gluten. Apart from enteropathy which is primary characteristic of the disease, it may include other extraintestinal organs [2]. Based on clinical, serological and histological variations, CD may be classified into two basic types: symptomatic and asymptomatic. Within symptomatic form of the disease, there are forms with classical and atypical (monosymptomatic and oligosymptomatic) clinical picture [3]. Classical form of the disease occurs primarily in infants and small children, while atypical are more common at older age $[1,2,3]$.

Classical histological feature of celiac enteropathy is villous atrophy, crypt hyperplasia, increased number of intraepithelial lymphocytes within lamina propria and lamina propria mononuclear cells [4]. In the presence of less severe histological changes (without villous atrophy), histological features become less specific for the diagnosis of CD [5]. The reasons why certain patients present with classical form of the disease while others remain completely asymptomatic, as well as whether the form of CD is related to severity of mucosal damage, is not entirely understood.

Therefore, this study was designed in order to determine whether there is any connection between histological abnormalities of small bowel mucosa and various forms of $\mathrm{CD}$ manifestation.

\section{Materials and Methods}

\subsection{Patients}

This study included children diagnosed with $\mathrm{CD}$ at the University Children's Clinic in Belgrade and Children's Department of the Subotica General Hospital from December 1, 2007 to December 1, 2012. University Children's Clinic in Belgrade contributed with 70 patients, while Children's Department of the Subotica General Hospital recruited 15 patients - a total of 85 patients. All patients were assigned to one of three groups. 
Classical CD (gastrointestinal symptoms and signs). This group included 41 children with predominant gastrointestinal abnormalities, i.e. chronic diarrhea (malabsorption syndrome).

Atypical CD (extraintestinal symptoms and signs). The second group of 32 children comprised patients whose symptoms were atypical (monosymptomatic with one and oligosymptomatic form with more than one symptom).

Asymptomatic CD (silent CD). The third group of 12 children included patients without symptoms and signs typical for $\mathrm{CD}$, in whom the condition was diagnosed based on characteristic histological changes on small bowel biopsy. Samples obtained by enterobiopsy that was performed routinely at esophagogastroduodenoscopy indicated for reasons other than $\mathrm{CD}$, as well as on evaluation of asymptomatic children with increased risk of CD.

The diagnosis of CD was based on histological analysis of multiple biopsy samples, positive $\mathrm{CD}$ specific antibodies, and HLA typing.

\subsection{Small Bowel Histology}

Three endoscopists performed the procedures with Olympus GIF-N30 and Olympus GIF-XP160 (Tokyo, Japan). Mucosa samples for histological evaluation were collected from the duodenal bulb (1 biopsy specimen) and from the second part or more distal parts of the duodenum (4 to 6 biopsy specimens) using FB-19K-1 and FB-24Q forceps. Each biopsy was oriented, fixed in $10 \%$ formalin and stained with hematoxylin and eosin. Biopsy samples from University Children's Clinic were examined by a expert GI tract pathologist (S.Z.), and biopsy samples from Subotica General Hospital were assessed and graded by a hospital pathologist (A.N.). In instances of uncertainty, biopsy samples from Subotica were sent to Institute of Pathology, Medical Faculty University of Belgrade. The grading was undertaken by a second investigator (S.Z.), and consensus was reached. Both investigators were blinded with respect to any clinical and serological data. Histologic evaluation was performed during the course of patient assessment and not specifically for this study.

The classification of the degree of the small bowel mucosa damage was made according to the Marsh criteria, modified by Oberhuber: Type 2 (hyperplastic changes), Type 3a (partial villous atrophy), Type 3b (subtotal villous atrophy) and Type 3c (total villous atrophy) [6]. We did not include patients with the lesser degree of damage (Type 1) in this study.
Based on extent of inflammatory changes, samples were also classified as uniform, where all samples showed comparable level of damage, and nonuniform, where various level of damage was present across different samples from the same patient.

\subsection{Serologic Markers}

Levels of immunoglobulin A (IgA) tissue transglutaminase (tTG) and total IgA were measured in all patients. A routine method was used for quantitative measurements of serum IgA levels. IgA tTG and IgG tTG were determined using commercial enzyme-linked immunosorbent assay (Orgentec Diagnostika, Mainz, Germany). A manufacturer's recommended level of $\geq 10 \mathrm{U} / \mathrm{mL}$ was used as cutoff value for a positive result.

\subsection{HLA Typing}

HLA DQB1* allele groups were determined using the Olerup SSP DQ low-resolution kit (Olerup SSP AB, Saltsjöbaden, Sweden)

\subsection{Statistical Analysis}

Categorical variables are presented as absolute and relative frequencies, whereas continuous variables are summarized as mean \pm SD. For evaluation of continuous data, chi-square test was used. The rejection of the null hypothesis was set at $5 \%(\mathrm{P}<0.05)$.

\section{Results}

Table 1 presents demographic characteristics of the 85 children.

Table 1. Demographic characteristics of patients with CD

\begin{tabular}{|l|l|}
\hline Age ( \pm SD) & 7 months -18 years $(6.59 \pm 5.93$ years $)$ \\
\hline Sex & \\
\hline Boys & $33(38.82 \%)$ \\
\hline Girls & $52(61.18 \%)$ \\
\hline Form of CD manifestation & \\
\hline Classical & $41(48.23 \%)$ \\
\hline Atypical & $32(37.64 \%)$ \\
\hline Asymptomatic & $12(14.12 \%)$ \\
\hline Atypical & \\
\hline Monosymptomatic & $24(28.23 \%)$ \\
\hline Oligosymptomatic & $8(9.41 \%)$ \\
\hline
\end{tabular}

Table 2 presents signs and symptoms in patients with monosymptomatic and oligosymptomatic form of CD with their absolute frequencies.

Table 2. Signs and symptoms in patients with monosymptomatic and oligosymptomatic form of CD

\begin{tabular}{|c|c|c|}
\hline \multicolumn{2}{|c|}{ Oligosymptomatic patients } \\
\hline Symptoms and clinical signs & No & Symptoms and clinical signs \\
\hline Short stature & 7 & Short stature, anemia \\
\hline Anemia & 6 & Short stature, malnutrition \\
\hline Chronic abdominal pain & 2 & Malnutrition, anemia \\
\hline Malnutrition & 2 & Short stature, anemia, constipation \\
\hline Alopecia & 2 & Malnutrition, chronic abdominal pain \\
\hline Constipation & 2 & Total \\
\hline Adiopathic thrombocytopenic purpura & 1 & 1 \\
\hline Aphthous stomatitis & 1 & \multicolumn{2}{|c}{8} \\
\hline Chronic fatigue & 1 & 24 \\
\hline Total & &
\end{tabular}


The majority of those with asymptomatic CD were diagnosed because of screening families in whom there was an individual with CD (7 cases) and patients with type 1 diabetes mellitus (3 cases), followed by and incidental finding on upper endoscopy (2 cases).
No patient showed exclusive bulbar abnormalities. Consistently with primary study objective, the correlation between severity of intestinal damage and form of CD manifestation was examined. Table 3 presents the distribution of mucosal damage in various forms of the disease.

Table 3. Severity of intestinal damage in children with classical, atypical monosymptomatic, atypical oligosymptomatic, and asymptomatic form of CD

\begin{tabular}{|c|c|c|c|c|c|}
\hline \multirow{2}{*}{ Form of CD } & \multicolumn{5}{|c|}{ Marsh type of small bowel mucosal damage } \\
\cline { 2 - 6 } & Type 2 & Type 3a & Type 3b & Type 3c & Total \\
\hline Classical & 0 & 6 & 18 & 3 & 41 \\
\hline Atypical oligosymp. & 0 & 1 & 4 & 8 & 8 \\
\hline Atypical monosymp. & 1 & 6 & 9 & 1 & 24 \\
\hline Asymptom. & 1 & 6 & 4 & 29 & 12 \\
\hline Total & 2 & 19 & 35 & \multicolumn{2}{c|}{8} \\
\hline
\end{tabular}

There was no significant difference in type of small bowel mucosal damage between forms of CD bowel mucosal damage between classical, atypical and asymptomatic form of the disease $(\mathrm{p}=0.079)$. Furthermore, there was no difference in type of small bowel mucosal damage between classical, atypical monosymptomatic, atypical oligosymptomatic and asymptomatic form of CD $(p=0.190)$. There was no difference in the degree of small manifestation.

This study also examined the correlation between uniformity of changes pertinent to intestinal damage and forms of CD manifestation. Figure 1 shows distribution of uniformity of changes across various forms of the disease.

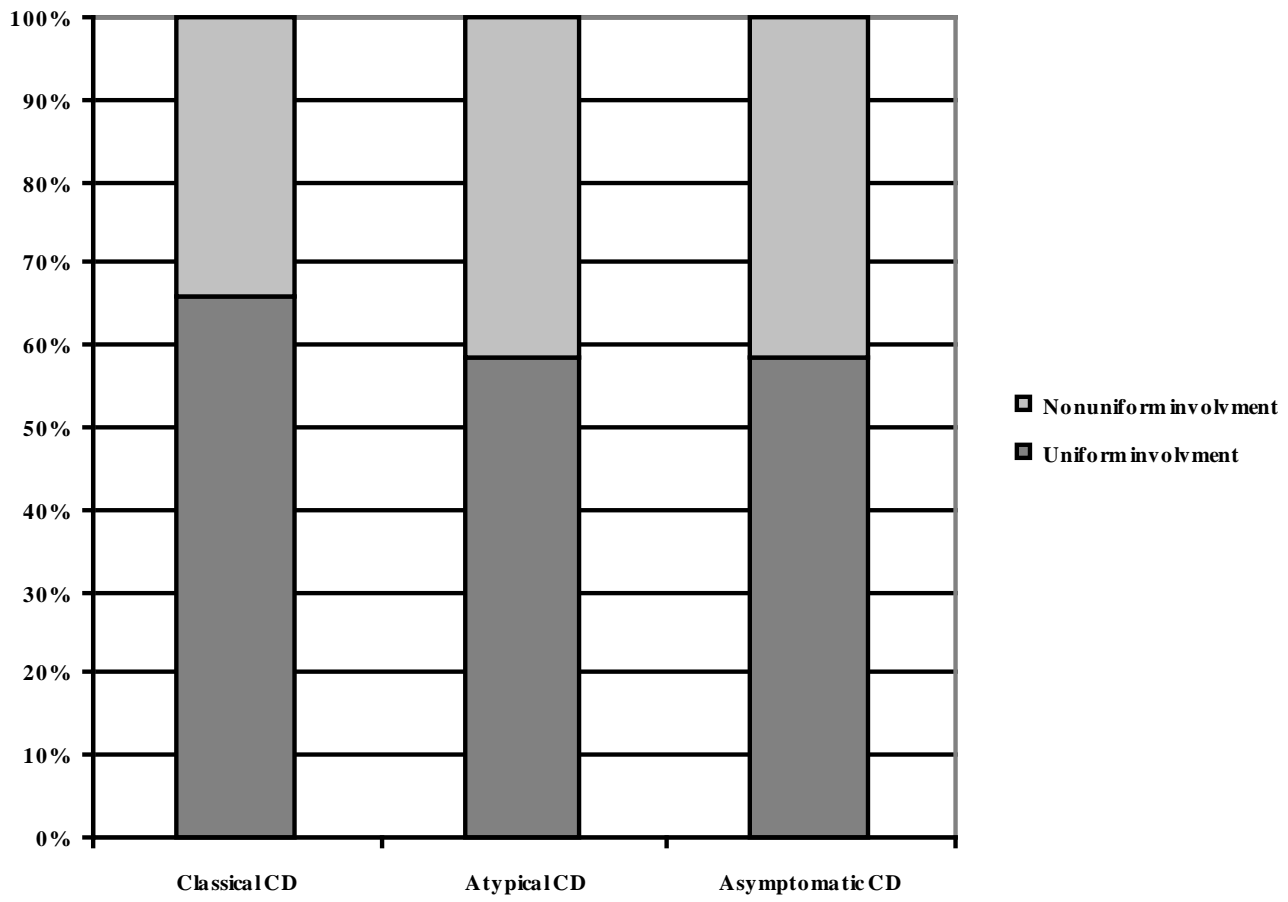

Figure 1. Uniformity (\%) of small bowel mucosal changes in various forms of CD manifestation

Among patients with atypical CD, 58.33\% (14/24) monosymptomatic patients had uniform mucosal involvement, while it was present in $75 \%$ (6/8) of oligosymptomatic patients.

The analysis of difference of uniformity of small bowel mucosal changes among classical, atypical and asymptomatic form of the disease yielded $p=0.882$. By comparing classical and atypical, classical and asymptomatic, atypical and asymptomatic, and mono- and oligosymptomatic form, no significant difference was observed in uniformity of changes between various pairs of forms of the disease $(\mathrm{p}=0.767, \mathrm{p}=0.633, \mathrm{p}=0.800$ and $\mathrm{p}=0.399$, respectively).

Furthermore, this study examined the correlation between level of mucosal damage and uniformity of changes. Table 4 shows empirical frequencies of uniformity of morphological damage across all forms of the disease.

Table 4. Uniformity of small bowel mucosal changes in various types of damage

\begin{tabular}{|c|c|c|c|c|c|}
\hline \multirow{2}{*}{ Uniformity of changes in all forms } & \multicolumn{5}{|c|}{ Marsh type of small bowel mucosal changes } \\
\cline { 2 - 6 } & Type 2 & Type 3a & Type 3b & Type 3c & All types \\
\hline Uniform changes & 2 & 8 & 20 & 24 & 54 \\
\hline Nonuniform changes & 0 & 11 & 15 & 5 & 31 \\
\hline Total & 2 & 19 & 35 & 29 & 85 \\
\hline
\end{tabular}


It may be concluded that uniform changes are significantly more common in patients with more severe small bowel mucosal damage, while nonuniform changes occur more commonly in patients with less severe mucosal damage $(p=0.017)$.

\section{Discussion}

The main finding of this study is that the degree of small bowel mucosal damage and uniformity of changes do not correlate with clinical forms of CD manifestation. The findings of other studies regarding this correlation are conflicting.

Two studies of children with CD yielded findings similar to those of this study [7], [8]. In a large study by Brar et al. that included 499 adult subjects, among patients with diarrhea, no severe level of villous atrophy was observed compared to patients with atypical/silent mode of presentation [9]. Authors argued that other factors that may contribute to diarrhea, such as abnormalities of serotonin metabolism. Our sample of 85 patients also showed no difference in type of small bowel mucosal damage among various forms of CD manifestation (classical, asymptomatic, mono- and oligosymptomatic).

Some authors believe that flattened mucosa seen in patients with CD does not always have to result in symptoms and that clinical presentation is more likely correlated with length of the affected portion of bowel [10]. Marsh also suggested the extent of enteropathy, i.e. length of involved small bowel, as an explanation for the presence of diarrheal syndrome [11]. Conversely, Murray et al. in their latest study from 2008 that included patients in whom the entire length of small bowel was analyzed using video capsule, found that the extent of visible enteropathy does not explain differences in clinical presentation [12].

In $\mathrm{CD}$, the level of histological damage in duodenum may be variable, even within the same fragment [13]. Bonamico et at. believe that the clinical presentation does not correlate with histological findings of patchiness [14]. Our data did not show any difference in uniformity of changes among various clinical forms either. On the other hand, progression of histological changes caused increased level of uniformity in more severe forms of enteropathy.

Tursi et al. have different opinion compared to the above mentioned [15]. Their study that included younger individuals with silent form of the disease showed increased frequency of mild and moderate histological mucosal changes. Other authors also believe that the degree of small bowel damage may correlate with clinical presentation of CD $[16,17]$. The attitude of the North American Society for Pediatric Gastroenterology, Hepatology and Nutrition (NASPHGAN) is that the milder changes and patchy lesions may be more likely when $\mathrm{CD}$ is diagnosed in patients with minimal or no symptoms [18].

Celiac disease features a spectrum of histological changes that develop over years through a dynamic process, starting from normal morphology, through inflammation, to crypt hyperplasia and villous atrophy. Clinical spectrum of CD includes patients with classical gastrointestinal symptoms and those with no complaints, who are diagnosed by screening of risk groups [19]. If the clinical manifestations of the disease would correlate with histological changes, milder forms would produce asymptomatic or atypical forms with less severe symptoms, while on the other end of the spectrum, villous atrophy would produce classical form of manifestation or atypical forms with apparent symptoms. Using this analogy, nonuniform changes should also be related to milder clinical forms, while clinical and biological features would depend on the area of noninvolved small bowel that would be able to compensate for a functional defect to a certain level. Our findings do not confirm this logical sequence of events. This is also suggested by several additional examples.

In the clinical practice, there are patients who have complaints even before histological changes develop on small bowel mucosa [20]. In up to $20 \%$ of patients, there is no detectable atrophy on duodenal biopsies after reintroduction of gluten in the diet, with persistence of serological markers (latent CD) [21]. Complaints may go away after administration of gluten free diet in spite of persistent enteropathy [22]. It is very likely that some other factors may influence development and manifestations of this multifactorial disease. It is known that homozygotes for DQB1*0201 alleles have more severe forms of CD with higher degree of villous atrophy, while patients with potential form of CD more commonly have HLA-DQB1*0302 and HLA-DQB1*0603 alleles $[23,24]$. Furthermore, environmental risk factors for the disease should be also taken into account, such as time of introduction of gluten in the diet, its amount, and breast feeding. $[25,26]$. Some authors think that small intestinal bacterial overgrowth secondary to motility disorder may also play a role in both symptom development and their persistence after introduction of gluten free diet [27].

\section{Conclusion}

Uniformity of enteropathy does not seem to depend on clinical form of the disease, while uniform small bowel mucosal damage appears to be more common in cases of more severe degrees of intestinal damage. The degree of intestinal damage is not related, nor does it determine the wide spectrum of clinical manifestations of CD.

\section{References}

[1] Walker AW, Durie PR, Hamilton RJ, Pediatric gastrointestinal disease, BC Decker Inc, Hamilton, 2000, 727-746.

[2] Van Heel DA, West J, Recent advances in coeliac disease, Gut, 55 (7), 1037-1046. Jul 2006.

[3] Polanco I, Celiac disease, J Pediatr Gastroenterol Nutr, 47 (S3-6). Aug 2008.

[4] Lionetti P, The enteropathy of celiac disease, $J$ Pediatr Gastroenterol Nutr, 34 (S18-21), May-Jun 2002.

[5] Biagi F, Bianchi PI, Campanella J, Badulli C, Martinetti M, Klersy C, Alvisi C, Luinetti O, Corazza GR, The prevalence and the causes of minimal intestinal lesions in patients complaining of symptoms suggestive of enteropathy: a follow-up study, J Clin Pathol, 61 (10),1116-1118. Oct 2008.

[6] Oberhuber G, Histopathology of celiac disease, Biomed Pharmacother, 54 (7), 368-372, Aug 2000.

[7] Weir DC, Glickman JN, Roiff T, Valim C, Leichtner AM, Variability of histopathological changes in childhood celiac disease, Am J of Gastroenterol, 105 (1), 207-212. Jan 2010. 
[8] Grzybowska-Chlebowczyk U, Wos H, Wiecek S, Kajor M, Szymanska M, Staszewska-Kwak A, Piatkowska M, Gołka D, Clinical picture of celiac disease in children, Pol Merkur Lekarski,18 (103), 49-53. Jan 2005.

[9] Brar P, Kwon GY, Egbuna II, Holleran S, Ramakrishnan R, Bhagat G, Green PH, Lack of correlation of degree of villous atrophy with severity of clinical presentation of coeliac disease, Dig Liver Dis, 39 (1), 26-29. Jan 2007.

[10] Dickson BC, Streutker CJ, Chetty R. Coeliac disease: an update for pathologists, J Clin Pathol, 59 (10), 1008-1016. Oct 2006.

[11] Marsh MN, Crowe PT, Morphology of the mucosal lesion in gluten sensitivity, Baillieres Clin Gastroenterol, 9 (2), 273-293. Jun 1995.

[12] Murray JA, Rubio-Tapia A, Van Dyke CT, Brogan DL Knipschield MA, Lahr B, Rumalla A, Zinsmeister AR, Gostout CJ, Mucosal atrophy in celiac disease: extent of involvement, correlation with clinical presentation, and response to treatment, Clin Gastroenterol Hepatol, 6 (2), 186-193. Feb 2008.

[13] Ravelli A, Villanacci V, Monfredini C, Martinazzi S, Grassi V, Manenti S, How patchy is patchy villous atrophy? Distribution pattern of histological lesions in the duodenum of children with celiac disease, Am J Gastroenterol, 105 (9), 2103-2110. Sep 2009.

[14] Bonamico M, Mariani P, Thanasi E, Ferri M, Nenna R, Tiberti C, Mora B, Mazzilli MC, Magliocca FM, Patchy villous atrophy of the duodenum in childhood celiac disease. $J$ Pediatr Gastroenterol Nutr, 38 (2), 204-207. Feb 2004.

[15] Tursi A, Brandimarte G, Giorgetti GM, Gigliobianco A, Endoscopic features of celiac disease in adults and their correlation with age, histological damage, and clinical form of the disease, Endoscopy, 34 (10), 787-792. Oct 2002.

[16] Taavela J, Kurppa K, Collin P, Lähdeaho ML, Salmi T, Saavalainen P, Haimila K, Huhtala $H$, Laurila K, Sievänen $H$, Mäki M, Kaukinen K, Degree of damage to the small bowel and serum antibody titers correlate with clinical presentation of patients with celiac disease, Clin Gastroenterol Hepatol, 11 (2), 166-171. Feb 2013.

[17] Dinler G, Atalay E, Kalayci AG, Celiac disease in 87 children with typical and atypical symptoms in Black Sea region of Turkey, World J Pediatr, 5 (4), 282-286. Nov 2009.
[18] Hill ID, Dirks MH, Liptak GS, Colletti RB, Fasano A, Guandalini S, Hoffenberg EJ, Horvath K, Murray JA, Pivor M, Seidman EG, Guideline for the diagnosis and treatment of celiac disease in children: recommendations of the North American Society for Pediatric Gastroenterology, Hepatology and Nutrition, J Pediatr Gastroenterol Nutr, 40 (1):1-19. Jan 2005.

[19] Radlovic N, Celiac disease, Srp Arh Celok Lek, 141 (1-2), 122-126. Jan-Feb 2013.

[20] Freeman HJ, Chopra A, Clandinin MT, Thomson AB, Recent advances in celiac disease, World J Gastroenterol, 17 (18), 22592272. May 2011.

[21] Matysiak-Budnik T, Malamut G, de Serre NP, Grosdidier E, Sequier S, Brousse N, Caillat-Zucman S, Cerf-Bensussan N, Schmitz J, Cellier C, Long-term follow-up of 61 coeliac patients diagnosed in childhood: evolution toward latency is possible on a normal diet, Gut, 56 (10), 1379-1386. Feb 2007.

[22] Kaukinen K, Peräaho M, Lindfors K, Partanen J, Woolley N, Pikkarainen P, Karvonen AL, Laasanen T, Sievänen H, Mäki M, Collin P, Persistent small bowel mucosal villous atrophy without symptoms in coeliac disease, Aliment Pharmacol Ther, 25 (10), 1237-1245. May 2007.

[23] Murray JA, Moore SB, Van Dyke CT, Lahr BD, Dierkhising RA, Zinsmeister AR, Melton LJ 3rd, Kroning CM, El-Yousseff M, Czaja AJ, HLA DQ gene dosage and risk and severity of celiac disease, Clin Gastroenterol Hepatol, 5 (12), 1406-1412. Oct 2007.

[24] Biagi F, Bianchi PI, Vattiato C, Marchese A, Trotta L, Badulli C, De Silvestri A, Martinetti M, Corazza GR, Influence of HLA-DQ2 and DQ8 on severity in celiac disease, J Clin Gastroenterol, 46 (1), 46-50. Jan 2012.

[25] Ascher H, Holm K, Kristiansson B, Mäki M, Different features of coeliac disease in two neighbouring countries, Arch Dis Child, 69 (3), 375-380. Sep 2003.

[26] Poddar U, Thapa BR, Singh K, Clinical features of celiac disease in Indian children: are they different from the West? J Pediatr Gastroenterol Nutr, 43 (3), 313-317. Sep 2006.

[27] Tursi A. Gastrointestinal motility disturbances in celiac disease, $J$ Clin Gastroenterol, 38 (8), 642-645. Sep 2004. 